Journal of Systems Science and Information

Feb., 2015, Vol. 3, No. 1, pp. 48-58

DOI: $10.1515 /$ jssi-2015-0048

\title{
A Comparison of Control Variate Methods for Pricing Interest Rate Derivatives in the LIBOR Market Model
}

\author{
Chenglong XU \\ Department of Mathematics, Tongji University, Shanghai 200092, China; Shanghai E-Institute of \\ Scientific Computing, Shanghai Normal University, Shanghai 200234, China \\ E-mail: clxu@tongji.edu.cn \\ Wei GUAN \\ Shanghai Pudong Development Bank Shanghai Branch, Shanghai 200120, China \\ E-mail:g123apple@163.com \\ Yijuan LIANG \\ College of Economics and Management, Southwest University, Chongqing 400715, China \\ E-mail: liangyijuan._0612@163.com
}

\begin{abstract}
This paper studies the control variate method for pricing interest rate derivatives driven by the LIBOR market model. Several control variates are constructed based on distinctive approximations for the LIBOR market model. Numerical results show the great efficiency of our methods. The idea in this paper can also be extended to price other interest rate derivatives under the LIBOR market model, such as Swaptions, Caps, some path dependent interest rate derivatives, and so forth.
\end{abstract}

Keywords LIBOR market model; Monte Carlo simulations; control variate

\section{Introduction}

In the past decades, many exotic interest rate derivatives have been developed to meet the needs of investors, such as Caps, Floors, Swaptions, and so forth. In these derivatives, interest rate is used not only for discounting as well as for defining the payoffs of the products. Therefore, choosing a suitable interest rate model is extremely significant. The LIBOR market model (LMM), which was developed by Brace, Gatarek and Musiela ${ }^{[1]}$, has become a standard model for the pricing of interest rate derivatives in recent years. Meanwhile, various details of its implementation are still being worked out, just like how to approximate the drift of the LMM given by a stochastic differential equation (SDE), for better approximations will lead to more accurate and efficient Monte Carlo simulations. Miltersen, Sandmann and Sondermann ${ }^{[2]}$ drove a unified structure of interest rates model and provided the closed form solutions for some rate derivatives. Brace, Gatarek and Musiela ${ }^{[1]}$ introduced the Frozen drift approximation. Glasserman and Zhao ${ }^{[3]}$ studied the arbitrage-free discretizational approximations of the LMM. Hull

Received March 13, 2014, accepted May 28, 2014

Supported by the National Natural Science Foundation of China (11171256), National Basic Research Program of China (973 Program) 2007CB 814903 and Shanghai Education Committee E-Research Subject E03004 
and White ${ }^{[4]}$, Jackel and Rebonato ${ }^{[5]}$ developed the methods of approximation for the LMM by high dimensional lognormal processes. Hunter, Jackel and Joshi ${ }^{[6]}$ approximated the drift of interest rate model over the mentioned long time steps to reduce the calculation considerably. Kurbanmuradov, Sabelfeld and Schoenmakers ${ }^{[7]}$ studied the neglecting the drift term approximation, the Log-normal approximation and others. Joshi and Stacey ${ }^{[8]}$ studied the tpc, mpc, capc and cami drift term of the LMM approximation methods. Siopacha and Teichmann ${ }^{[9]}$, Papapantoleon and Siopacha ${ }^{[10]}$ developed the strong Taylor approximation for the drift term of the LMM while Papapantoleon and Skovmand ${ }^{[11]}$ studied the Picard approximation for it.

On the other hand, it is not easy to solve explicitly the SDE corresponding to the LMM in pricing the interest rate derivatives, therefore it requires the use of some numerical methods to solve them. Joshi and Stacey ${ }^{[8]}$ and some others mentioned above used Monte Carlo Simulations in the pricing of LIBOR rate derivatives. Furthermore, Pelsser, Jong, Driessen ${ }^{[12]}$ used Monte Carlo method to price Caps within the LMM. But they had no discussion about acceleration method to improve the Monte Carlo simulation efficiency and reduce the variance in the simulations, the latter plays key role in increasing the efficiency of simulations.

The method of control variate is one of the most widely used variance reduction techniques and so it is among the most effective applicable techniques for improving the efficiency of Monte Carlo simulations. It exploits information about the errors in estimates of known quantities to reduce the errors in an estimate of an unknown quantity. To illustrate the method of control variate, we consider the problem of estimating the expectation of a random variable $V$, the discounted payoff of a derivative, such as caplet, Caps, Swaption, etc. Let $V_{1}, V_{2}, \cdots, V_{m}$ be the outputs from $m$ replications and suppose that each $V_{i}$ is independent and identically distributed (i.i.d.). The estimator of the price is of the average

$$
\bar{V}=\frac{V_{1}+V_{2}+\cdots+V_{m}}{m} .
$$

Suppose that on each simulation there is another output $X_{j}$ along with $V_{j}$ and the pairs $\left(V_{j}, X_{j}\right)$ are i.i.d.. Meanwhile, the expectation $E\left[X_{j}\right]=E[X]$ is assumed known. Then for any fixed coefficient $b$ we can calculate

$$
V_{j}(b)=V_{j}-b\left(X_{j}-E[X]\right)
$$

from the $j$ th simulation. Therefore the control variate estimator of the price is given by

$$
\bar{V}(b)=\frac{1}{m} \sum_{j=1}^{m}\left(V_{j}-b\left(X_{j}-E[X]\right)\right) .
$$

It is obvious that $V(b)=V-b(X-E[X])$ is an unbiased estimator of $V$ and its variance is

$$
\operatorname{Var}(V(b))=\operatorname{Var}(V)+b^{2} \operatorname{Var}(X)-2 b \sqrt{\operatorname{Var}(V) \operatorname{Var}(X)} \rho_{X, V} .
$$

To minimize the variance, the optimal coefficient $b^{*}=\frac{\operatorname{Cov}[V, X]}{\operatorname{Var}[X]}$ is selected and then

$$
\operatorname{Var}\left(V\left(b^{*}\right)\right)=\left(1-\rho_{X, V}^{2}\right) \operatorname{Var}(V) .
$$

Consequently, we should choose proper control variate $X$ which has high correlation with $V$ to reduce the variance. 
In this paper, we will use the control variate method to improve the efficiency of Monte Carlo simulation in evaluating the value of some interest rate derivatives in the LIBOR market model. Firstly, we will present three approximation methods which were widely used in practice and introduce two new ones based on direct simulations. Secondly, we will combine these approximations with control variate method to reduce the variance of simulation estimates and increase the efficiency of Monte Carlo simulations. We focus on the LIBOR market model for a discrete set of tenors given by a stochastic differential equation (SDE) in the terminal bond measure as developed in [13]. We will compare the efficiencies of simulations in the valuation of the LIBOR derivatives for different simulation methods based on the different control variates.

The rest of the paper is organized as follows. In Section 2, we recall three approximation methods of the LIBOR market model, the neglecting the drift term approximation, the frozen drift approximation and the Log-normal approximation, which had been studied in some literatures and propose two new ones based on direct simulations, the optimal linear function approximation and the optimal piecewise function approximation. In Section 3, we construct different control variate approximates, based on the approximation results in Section 2. As an example, we will demonstrate the simulation efficiency for evaluating the value of Caplets by the method of control variate acceleration. We will also analyze the simulation results with different control variates as well as various parameters in LIBOR market model. The idea in this paper may also applicable for pricing other interest rate derivatives base on the LIBOR market model.

\section{Approximations of LIBOR Market Model}

For a given tenor structure $0=T_{0}<T_{1}<\cdots<T_{n}<T_{n+1}$, we consider a LIBOR market model for the forward LIBOR process $L_{i}(t)$ in the terminal bond numeraire $Q^{n+1}$ (see, e.g., [21])

$$
\mathrm{d} L_{i}(t)=\mu_{i}(t) L_{i}(t) \mathrm{d} t+L_{i}(t) \sigma_{i}^{\mathrm{T}}(t) \mathrm{d} W^{(n+1)}(t), t \in\left[T_{0}, T_{i}\right], i=1,2, \cdots, n
$$

where

$$
\mu_{i}(t)=-\sum_{l=i+1}^{n} \frac{\delta_{l} L_{l}(t) \sigma_{i}^{\mathrm{T}}(t) \sigma_{l}(t)}{1+\delta_{l} L_{l}(t)}
$$

$L_{i}(t)$ is defined in the interval $\left[T_{0}, T_{i}\right], \delta_{i}=T_{i+1}-T_{i}(i=1,2, \cdots, n)$ and $\sigma_{i, j}(t)(j=1,2, \cdots, d)$ are given deterministic functions also defined in $\left[T_{0}, T_{i}\right], \sigma_{i}(t)=\left(\sigma_{i, 1}(t), \sigma_{i, 2}(t), \cdots, \sigma_{i, d}(t)\right)^{\mathrm{T}}$. $W_{1}(t), W_{2}(t), \cdots, W_{d}(t)$ are Brown motions independent with each other, $W^{(n+1)}(t)=\left(W_{1}(t)\right.$, $\left.W_{2}(t), \cdots, W_{d}(t)\right)^{\mathrm{T}}\left(t \in\left[T_{0}, T_{n}\right]\right)$ is a standard $d$-dimensional Wiener process under $Q^{n+1}$. When $d=1$, (1) becomes a one-factor model with $\sigma_{i}(t)=\sigma_{i, 1}(t), W^{(n+1)}(t)=W_{1}(t)$.

Next, we present several approximation methods. Among them, the neglecting the drift term approximation is studied by lots of authors, the frozen drift approximation was first introduced by Brace, Gatarek and Musiela ${ }^{[1]}$, the Log-normal approximation was developed by Kurbanmuradov, Sabelfeld and Schoenmakers ${ }^{[7]}$.

\section{Method 1: Neglecting the drift term approximation}

Neglecting the random drift term in (1), we have that

$$
\mathrm{d} L_{i}^{(1)}(t)=L_{i}^{(1)}(t) \sigma_{i}^{\mathrm{T}}(t) \mathrm{d} W^{(n+1)}(t), \quad t \in\left[T_{0}, T_{i}\right], i=1,2, \cdots, n
$$


with $\mu_{i}^{(1)}(t)=0$. So the solution of (1) can be approximated by

$$
L_{i}^{(1)}(t)=L_{i}(0) \exp \left(-\frac{1}{2} \int_{0}^{t} \sigma_{i}^{\mathrm{T}}(s) \sigma_{i}(s) \mathrm{d} s+\int_{0}^{t} \sigma_{i}^{\mathrm{T}}(s) \mathrm{d} W^{(n+1)}(s)\right)
$$

\section{Method 2: Frozen drift approximation}

Replacing the random drift term in (1) by their deterministic initial values, (1) can be approximated by

$$
\mathrm{d} L_{i}^{(2)}(t)=\mu_{i}^{(2)}(t) L_{i}^{(2)}(t) \mathrm{d} t+L_{i}^{(2)}(t) \sigma_{i}^{\mathrm{T}}(t) \mathrm{d} W^{(n+1)}(t), \quad t \in\left[T_{0}, T_{i}\right], i=1,2, \cdots, n
$$

where

$$
\mu_{i}^{(2)}(t)=-\sum_{l=i+1}^{n} \frac{\delta_{l} L_{l}(0) \sigma_{i}^{\mathrm{T}}(t) \sigma_{l}(t)}{1+\delta_{l} L_{l}(0)} \mathrm{d} t
$$

Then the solution of (5) can be given by

$$
L_{i}^{(2)}(t)=L_{i}(0) \exp \left(\int_{0}^{t}\left(\mu_{i}^{(2)}(s)-\frac{1}{2} \sigma_{i}^{\mathrm{T}}(s) \sigma_{i}(s)\right) \mathrm{d} s+\int_{0}^{t} \sigma_{i}^{\mathrm{T}}(s) \mathrm{d} W^{(n+1)}(s)\right)
$$

The above approximation was first proposed by Brace et al ${ }^{[1]}$.

\section{Method 3: Log-normal approximation}

Let $f$ be a function defined by $f(x)=\frac{\delta_{i} x}{1+\delta_{i} x}$ and $Y_{i}(t)=f\left(L_{i}(t)\right)(i=1,2, \cdots, n)$. By (1) and using Itô formula, we find that $Y_{i}(t)$ satisfies the following SDE

$$
\begin{aligned}
\mathrm{d} Y_{i}(t)= & \left(\mu_{i}(t) f^{\prime}\left(L_{i}(t)\right) L_{i}(t)+\frac{1}{2} f^{\prime \prime}\left(L_{i}(t)\right) L_{i}^{2}(t) \sigma_{i}^{\mathrm{T}}(t) \sigma_{i}(t)\right) \mathrm{d} t \\
& +f^{\prime}\left(L_{i}(t)\right) L_{i}(t) \sigma_{i}^{\mathrm{T}}(t) \mathrm{d} W^{(n+1)}(t), \\
Y_{i}(0)= & f\left(L_{i}(0)\right) .
\end{aligned}
$$

The first term of the Picard Iteration to the solution of above SDE is $Y_{i}^{(0)}(t) \approx Y_{i}(0)=$ $\frac{\delta_{i} L_{i}(0)}{1+\delta_{i} L_{i}(0)}$, which can be regarded as the approximation in the method 2. The second term of the Picard Iteration to the solution of above SDE is

$$
\begin{aligned}
Y_{i}^{(1)}(t) \approx & f\left(L_{i}(0)\right)+\mu_{i}(0) f^{\prime}\left(L_{i}(0)\right) L_{i}(0) t+\frac{1}{2} f^{\prime \prime}\left(L_{i}(0)\right) L_{i}^{2}(0) \int_{0}^{t} \sigma_{i}^{\mathrm{T}}(s) \sigma_{i}(s) \mathrm{d} s \\
& +f^{\prime}\left(L_{i}(0)\right) L_{i}(0) \int_{0}^{t} \sigma_{i}^{\mathrm{T}}(s) \mathrm{d} W^{(n+1)}(s) \\
= & \frac{\delta_{i} L_{i}(0)}{1+\delta_{i} L_{i}(0)}+\mu_{i}(0) \frac{\delta_{i} L_{i}(0)}{\left(1+\delta_{i} L_{i}(0)\right)^{2}} t-\frac{\delta_{i}^{2}}{\left(1+\delta_{i} L_{i}(0)\right)^{3}} L_{i}^{2}(0) \int_{0}^{t} \sigma_{i}^{\mathrm{T}}(s) \sigma_{i}(s) \mathrm{d} s \\
& +\frac{\delta_{i} L_{i}(0)}{\left(1+\delta_{i} L_{i}(0)\right)^{2}} \int_{0}^{t} \sigma_{i}^{\mathrm{T}}(s) \mathrm{d} W^{(n+1)}(s)
\end{aligned}
$$

Replacing $\frac{\delta_{l} L_{l}(t)}{1+\delta_{l} L_{l}(t)}$ in expression of $\mu_{i}(t)$ in $(2)$ by $Y_{l}^{(1)}(t)$, we get

$$
\mu_{i}(t) \approx \mu^{(3)}(t)=-\sum_{l=i+1}^{n} Y_{l}^{(1)}(t) \sigma_{i}^{\mathrm{T}}(t) \sigma_{l}(t)
$$


Thus the solution of (1) can be approximated by

$$
L_{i}^{(3)}(t)=L_{i}(0) \exp \left(\int_{0}^{t}\left(\mu_{i}^{(3)}(s)-\frac{1}{2} \sigma_{i}^{\mathrm{T}}(s) \sigma_{i}(s)\right) \mathrm{d} s+\int_{0}^{t} \sigma_{i}^{\mathrm{T}}(s) \mathrm{d} W^{(n+1)}(s)\right)
$$

Next, we will propose two new approximations for the random drift function $\mu_{i}(t)$ by $\mu^{(4)}(t)$ and $\mu^{(5)}(t)$ respectively, based on direct simulations.

\section{Method 4: Optimal linear function approximation based on direct simulations}

Divide each interval $\left[T_{i}, T_{i+1}\right]$ into $K$ equal parts with mesh size $\triangle t=t_{k+1}-t_{k}$. Based on the Euler discretization for $\operatorname{SDE}(1)$, generate a standard normal vector $Z=\left(Z_{1}, Z_{2}, \cdots, Z_{n}\right)$ each $Z_{i}(i=1,2, \cdots, n)$ is a standard normal random variable and independent with each other. For the $j$ th simulation, let

$$
L_{i}^{j}\left(t_{k+1}\right)=L_{i}^{j}\left(t_{k}\right)+\mu_{i}^{j}\left(t_{k}\right) L_{i}^{j}\left(t_{k}\right) \triangle t+L_{i}^{j}\left(t_{k}\right) \sigma_{i}^{\mathrm{T}}\left(t_{k}\right) \sqrt{\triangle t} Z^{j}
$$

where

$$
\mu_{i}^{j}\left(t_{k}\right)=-\sum_{l=i+1}^{n} \frac{\delta_{l} L_{l}^{j}\left(t_{k}\right) \sigma_{i}^{\mathrm{T}}\left(t_{k}\right) \sigma_{l}\left(t_{k}\right)}{1+\delta_{l} L_{l}^{j}\left(t_{k}\right)} .
$$

Let $m$ be the simulation times and

$$
\mu_{i}^{(4)}(t)=a_{i}+b_{i} t, \quad J=\sum_{j=1}^{m} \sum_{i=1}^{n} \sum_{k=1}^{i K}\left(\mu_{i}^{j}\left(t_{k}\right)-\mu_{i}^{(4)}\left(t_{k}\right)\right)^{2}
$$

Choosing $a_{i}, b_{i}(i=1,2, \cdots, n)$, such that $\left(a_{1}, b_{1}, \cdots, a_{n}, b_{n}\right)=\arg \min _{a_{i}, b_{i}} J\left(a_{1}, b_{1}, \cdots, a_{n}, b_{n}\right)$. Then

$$
\frac{\partial J}{\partial a_{i}}=0, \quad \frac{\partial J}{\partial b_{i}}=0, \quad i=1,2, \cdots, n,
$$

lead to

$$
\begin{aligned}
a_{i}= & \frac{\sum_{j=1}^{m} \sum_{k=1}^{i K} \mu_{i}^{j}\left(t_{k}\right)\left(i K \sum_{k=1}^{i K} t_{k}^{2}-\left(\sum_{k=1}^{i K} t_{k}\right)^{2}\right)-\sum_{k=1}^{i K} t_{k}\left(i K \sum_{j=1}^{m} \sum_{k=1}^{i K} \mu_{i}^{j}\left(t_{k}\right) t_{k}-\sum_{k=1}^{i K} t_{k} \sum_{j=1}^{m} \sum_{k=1}^{i K} \mu_{i}^{j}\left(t_{k}\right)\right)}{m i K\left(i K \sum_{k=1}^{i K} t_{k}^{2}-\left(\sum_{k=1}^{i K} t_{k}\right)^{2}\right)} \\
b_{i}= & \frac{i K \sum_{j=1}^{m} \sum_{k=1}^{i K} \mu_{i}^{j}\left(t_{k}\right) t_{k}-\sum_{k=1}^{i K} t_{k} \sum_{j=1}^{m} \sum_{k=1}^{i K} \mu_{i}^{j}\left(t_{k}\right)}{m\left(i K \sum_{k=1}^{i K} t_{k}^{2}-\left(\sum_{k=1}^{i K} t_{k}\right)^{2}\right)}
\end{aligned}
$$

So (1) can be approximated by equation

$$
\mathrm{d} L_{i}^{(4)}(t)=\mu_{i}^{(4)}(t) L_{i}^{(4)}(t) \mathrm{d} t+L_{i}^{(4)}(t) \sigma_{i}^{\mathrm{T}}(t) \mathrm{d} W^{(n+1)}(t), \quad t \in\left[T_{0}, T_{i}\right], i=1,2, \cdots, n
$$

and the corresponding solution of (1) can by approximated by

$$
L_{i}^{(4)}(t)=L_{i}(0) \exp \left(\int_{0}^{t}\left(\mu_{i}^{(4)}(s)-\frac{1}{2} \sigma_{i}^{\mathrm{T}}(s) \sigma_{i}(s)\right) \mathrm{d} s+\int_{0}^{t} \sigma_{i}^{\mathrm{T}}(s) \mathrm{d} W^{(n+1)}(s)\right)
$$


Method 5: Optimal piecewise function approximation based on direct simulations

We define piecewise functions $\mu_{i}^{(5)}(t)$, such that $\mu_{i}^{(5)}\left(t_{k}\right)=a_{i, k}$ for $t \in\left[t_{k-1}, t_{k}\right]$, and let $a=\left(a_{11}, a_{21}, a_{22} \cdots, a_{n n}\right)$

$$
J(a)=\sum_{j=1}^{m} \sum_{i=1}^{n} \sum_{k=1}^{i K}\left(\mu_{i}^{j}\left(t_{k}\right)-\mu_{i}^{(5)}\left(t_{k}\right)\right)^{2}
$$

Choosing $a_{i, k}$ such that $a=\arg \min _{a_{i, k}} J(a)$, then

$$
\frac{\partial J}{\partial a_{i, k}}=0
$$

lead to

$$
a_{i, k}=\frac{1}{m} \sum_{j=1}^{m} \mu_{i}^{j}\left(t_{k}\right), \quad k=1,2, \cdots, i K .
$$

Thus the solution of (1) can be approximated by

$$
L_{i}^{(5)}(t)=L_{i}(0) \exp \left(\int_{0}^{t}\left(\mu_{i}^{(5)}(s)-\frac{1}{2} \sigma_{i}^{\mathrm{T}}(s) \sigma_{i}(s)\right) \mathrm{d} s+\int_{0}^{t} \sigma_{i}^{\mathrm{T}}(s) \mathrm{d} W^{(n+1)}(s)\right)
$$

\section{Numerical Results for Pricing of Caplets}

Due to a Cap is a collection of caplets and that each caplet may be viewed as a call option on a forward rate, so we primarily analyze simulation results for each caplet next. In order to evaluate the value of caplet, different Monte Carlo simulation methods are used based on the different control variates.

The value of a caplet $_{i}$, defined in the accrual period $\left[T_{i}, T_{i+1}\right] \quad(i=1,2, \cdots, n-1)$, can be represented in the numeraire $Q^{n+1}$ by (see, e.g., [14])

$$
V_{\text {caplet }_{i}}=B_{n+1}(0) E\left[\frac{\delta_{i}\left(L_{i}\left(T_{i}\right)-K\right)^{+}}{B_{n+1}\left(T_{i+1}\right)}\right]
$$

where $B_{n+1}(t)$ is the time $t$ price of zero-coupon bond with maturing at $T_{n+1}\left(0 \leq t \leq T_{n+1}\right)$,

$$
B_{n+1}(0)=\prod_{l=0}^{n} \frac{1}{1+\delta_{l} L_{l}(0)}, \quad B_{n+1}\left(T_{i+1}\right)=\prod_{l=i+1}^{n} \frac{1}{1+\delta_{l} L_{l}\left(T_{i+1}\right)} .
$$

The algorithm for the valuation of a caplet with control variate technique is then given as follows.

i) Divide each interval $\left[T_{i}, T_{i+1}\right]$ into $K$ equal parts with mesh size $\triangle t=t_{k+1}-t_{k}$. Based on the Euler discretization for $\operatorname{SDE}(1)$, generate a standard normal vector $Z=\left(Z_{1}, Z_{2}, \cdots, Z_{d}\right)$, $Z_{i}(i=1,2, \cdots, d)$ are standard normal random variables and independent with each other,

$$
L_{i}^{j}\left(t_{k+1}\right)=L_{i}^{j}\left(t_{k}\right)-\sum_{l=i+1}^{n} \frac{\delta_{l} L_{l}^{j}\left(t_{k}\right) \sigma_{i}^{\mathrm{T}}\left(t_{k}\right) \sigma_{l}\left(t_{k}\right) L_{i}^{j}\left(t_{k}\right) \triangle t}{1+\delta_{l} L_{l}^{j}\left(t_{k}\right)}+L_{i}^{j}\left(t_{k}\right) \sigma_{i}^{\mathrm{T}}\left(t_{k}\right) \sqrt{\triangle t} Z^{j, k}
$$

then the $j$ th $(j=1,2, \cdots, m)$ replication of the LIBOR value $L_{i}(t)$ is attained, and the price of caplet $_{i}$ is given by

$$
V_{\text {caplet }_{i}}^{j}=B_{n+1}^{j}(0) \frac{\delta_{i}\left(L_{i}^{j}\left(T_{i}\right)-K\right)^{+}}{B_{n+1}^{j}\left(T_{i+1}\right)}
$$


ii) Let

$$
\mathrm{d} \widehat{L}_{i}(t)=\widehat{\mu}_{i}(t) \widehat{L}_{i}(t) \mathrm{d} t+\widehat{L}_{i}(t) \sigma_{i}^{\mathrm{T}}(t) \mathrm{d} W
$$

with deterministic drift functions $\widehat{\mu}_{i}(t)$, which will replaced by $\mu_{i}^{(1)}(t), \cdots, \mu_{i}^{(5)}(t)$ respectively. Then for any $k \leq i-1$,

$$
\widehat{L}_{i}^{j}\left(t_{k+1}\right)=\widehat{L}_{i}^{j}\left(t_{k}\right) \exp \left(\left(\widehat{\mu_{i}}\left(t_{k}\right)-\frac{1}{2} \sigma_{i}^{\mathrm{T}}\left(t_{k}\right) \sigma_{i}\left(t_{k}\right)\right) \triangle t+\sigma_{i}^{\mathrm{T}}\left(t_{k}\right) \sqrt{\triangle t} Z^{j, k}\right),
$$

and

$$
V_{\text {caplet }_{i}}^{j}=\widehat{B}_{n+1}(0) \frac{\delta_{i}\left(\widehat{L}_{i}^{j}\left(T_{i}\right)-K\right)^{+}}{\widehat{B}_{n+1}^{j}\left(T_{i+1}\right)}
$$

where

$$
\widehat{B}_{n+1}^{j}\left(T_{i+1}\right)=\prod_{l=0}^{n} \frac{1}{1+\delta_{l} \widehat{L}_{l}^{j}\left(T_{i+1}\right)}, \quad \widehat{B}_{n+1}(0)=\prod_{l=i+1}^{n} \frac{1}{1+\delta_{l} L_{l}(0)} .
$$

iii) Set

$$
V^{j}(b)=V_{\text {caplet }_{i}}^{j}-b\left(V_{\text {caplet }_{i}}^{j}-E\left[V_{\text {caplet }_{i}}\right]\right)
$$

then

$$
E\left[V_{\text {caplet }_{i}}\right]=\delta_{i} \widehat{B}_{n+1}(0) E\left[\left(\widehat{L}_{i}\left(T_{i}\right)-K\right)^{+}\right] E\left[\prod_{l=i+1}^{n}\left(1+\delta_{l} \widehat{L}_{l}\left(T_{i+1}\right)\right)\right] .
$$

It is easy to see that

$$
\begin{aligned}
& E\left[\prod_{l=i+1}^{n}\left(1+\delta_{l} \widehat{L}_{l}\left(T_{i+1}\right)\right)\right]=\prod_{l=i+1}^{n}\left(1+\delta_{l} \widehat{L}_{l}(0) \exp \left(\widehat{\mu}_{k}\left(T_{i+1}\right) T_{i+1}\right)\right), \\
& \delta_{i} \widehat{B}_{n+1}(0) E\left[\left(\widehat{L}_{i}\left(T_{i}\right)-K\right)^{+}\right]=B C\left(\widehat{L}_{k}(0), \widetilde{\sigma}_{i}, T_{i}, K, \delta_{i} \widehat{B}_{n+1}(0)\right),
\end{aligned}
$$

where

$$
\begin{aligned}
& \tilde{\sigma}_{i}=\sqrt{\sigma_{i, 1}^{2}\left(T_{i}\right)+\sigma_{i, 2}^{2}\left(T_{i}\right)+\cdots+\sigma_{i, d}^{2}\left(T_{i}\right)} \\
& B C(F, \sigma, T, K, b)=b\left(F \Phi\left(\frac{\log (F / K)+\sigma^{2} T / 2}{\sigma \sqrt{T}}\right)-K \Phi\left(\frac{\log (F / K)-\sigma^{2} T / 2}{\sigma \sqrt{T}}\right)\right) .
\end{aligned}
$$

iv) The control variate estimate of the price of the caplet $_{i}$ is finally obtained from the mean of $m$ replications

$$
\bar{V}(b)=\frac{1}{m} \sum_{j=1}^{m}\left(V_{\text {caplet }_{i}}^{j}-b\left(V_{\text {caplet }_{i}}^{j}-E\left[V_{\text {caplet }_{i}}\right]\right)\right)
$$

In practice, $b=\frac{\operatorname{cov}\left(V_{\text {caplet }_{i},}, V_{\text {caplet }_{i}}\right)}{\operatorname{var}\left(V_{\text {caplet }_{i}}\right)}$ is given by

$$
\begin{aligned}
& \widehat{b}=\sum_{j=1}^{m} \frac{\left(V_{\text {caplet }_{i}}^{j}-\bar{V}_{\text {caplet }_{i}}\right)\left(V_{\text {capplet }_{i}}^{j}-\bar{V}_{\text {caplet }_{i}}\right)}{\left(V_{\text {câplet }_{i}}^{j}-\bar{V}_{\text {caplet }_{i}}\right)^{2}} \\
& \bar{V}_{\text {caplet }_{i}}=\frac{1}{m} \sum_{j=1}^{m} V_{\text {caplet }_{i}}^{j}, \quad \bar{V}_{\text {câplet }_{i}}=\frac{1}{m} \sum_{j=1}^{m} V_{\text {caplet }_{i}}^{j}
\end{aligned}
$$

where $V_{\text {caplet }_{i}}^{j}$ and $V_{\text {caplet }_{i}}^{j}$ are calculated by (19) and (20), respectively. 
Now, we present some computation results based on the control variate method illustrated above under the multi-factor model. Taking the initial LIBOR value as $L_{i}(0)=0.05$, the strike price as $K=0.05$ and $\sigma_{i, 1}(t)=\cdots=\sigma_{i, d}(t)=\sigma(d=n, i=1,2, \cdots, n)$.

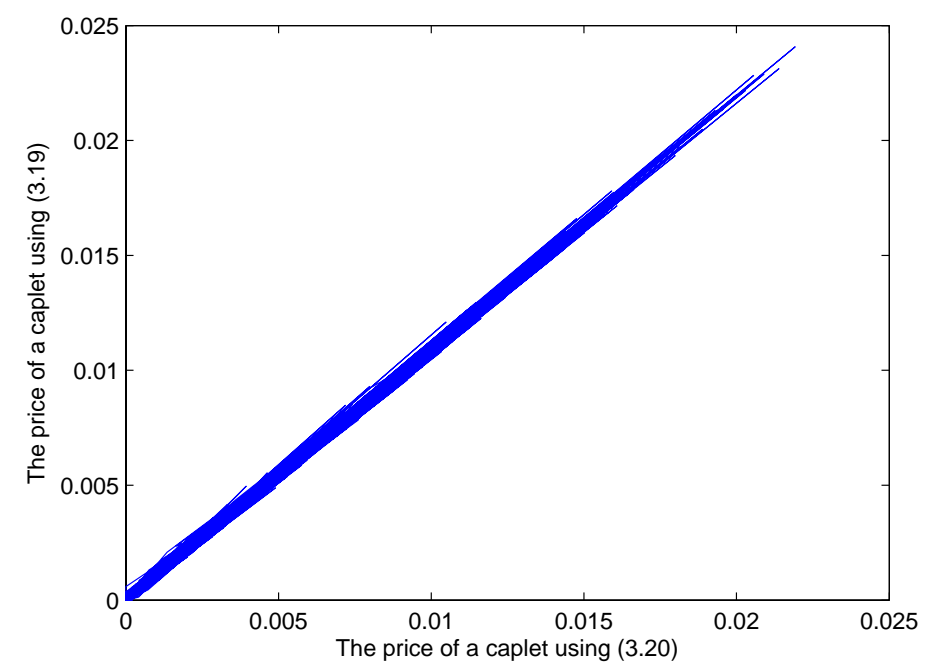

Figure 1 Scatter plots of values of caplet and control variate for $n=2, L_{i}(0)=$ $0.05, K=0.05, \sigma=0.2, \triangle T=0.5, \triangle t=\frac{1}{30}, m=10^{4}$

Firstly, we present the simulation results for one caplet and the tenor structure is $0=$ $T_{0}<T_{1}<T_{2}<T_{3}$, the terminal numeraire is $Q^{3}$. Figure 1 shows scatter plots of simulated values of $B_{3}(0) \frac{\delta_{1}\left(L_{1}\left(T_{1}\right)-K\right)^{+}}{B_{3}\left(T_{2}\right)}$ with approximated values $\widehat{B}_{3}(0) \frac{\delta_{1}\left(L_{1}^{(s)}\left(T_{1}\right)-K\right)^{+}}{\widehat{B}_{3}\left(T_{2}\right)}$ (serving as the corresponding control variates for $s=1,2, \cdots, 5)$ in the case of $n=2, \sigma=0.2$, mesh size $\triangle t=\frac{1}{30}, \delta_{i}=\triangle T=T_{i+1}-T_{i}=0.5(i=1,2,3)$, simulation times $m=10^{4}$. The plot shows the strong correlation between the simulated caplet values and the approximated caplet values of different control variate and their correlations are all more than 0.9995 .

Table 1 Simulation results for different control variates and simulation paths with $\Delta t=\frac{1}{30}$

\begin{tabular}{ccccccc}
\hline$m$ & Error & Error1 & Error2 & Error3 & Error4 & Error5 \\
\hline $1 \times 10^{3}$ & $2.1 \times 10^{-6}$ & $5.0 \times 10^{-8}$ & $5.3 \times 10^{-8}$ & $5.3 \times 10^{-8}$ & $5.4 \times 10^{-8}$ & $5.3 \times 10^{-8}$ \\
$5 \times 10^{3}$ & $4.7 \times 10^{-7}$ & $1.1 \times 10^{-8}$ & $1.1 \times 10^{-8}$ & $1.1 \times 10^{-8}$ & $1.1 \times 10^{-8}$ & $1.1 \times 10^{-8}$ \\
$1 \times 10^{4}$ & $2.3 \times 10^{-7}$ & $5.2 \times 10^{-9}$ & $5.6 \times 10^{-9}$ & $5.6 \times 10^{-9}$ & $5.7 \times 10^{-9}$ & $5.6 \times 10^{-9}$ \\
$5 \times 10^{4}$ & $4.7 \times 10^{-8}$ & $1.1 \times 10^{-9}$ & $1.2 \times 10^{-9}$ & $1.2 \times 10^{-9}$ & $1.2 \times 10^{-9}$ & $1.1 \times 10^{-9}$ \\
\hline
\end{tabular}

Table 2 Simulation results for different control variates and simulation paths with $\Delta t=\frac{1}{300}$

\begin{tabular}{ccccccc}
\hline$m$ & Error & Error1 & Error2 & Error3 & Error4 & Error5 \\
\hline $1 \times 10^{3}$ & $2.3 \times 10^{-6}$ & $2.0 \times 10^{-8}$ & $1.7 \times 10^{-8}$ & $1.7 \times 10^{-8}$ & $5.4 \times 10^{-8}$ & $5.3 \times 10^{-8}$ \\
$5 \times 10^{3}$ & $4.6 \times 10^{-7}$ & $4.0 \times 10^{-9}$ & $3.5 \times 10^{-9}$ & $3.4 \times 10^{-9}$ & $3.7 \times 10^{-9}$ & $3.5 \times 10^{-9}$ \\
$1 \times 10^{4}$ & $2.3 \times 10^{-7}$ & $2.0 \times 10^{-9}$ & $1.7 \times 10^{-9}$ & $1.7 \times 10^{-9}$ & $1.8 \times 10^{-9}$ & $1.7 \times 10^{-9}$ \\
$5 \times 10^{4}$ & $4.7 \times 10^{-8}$ & $4.0 \times 10^{-10}$ & $3.5 \times 10^{-10}$ & $3.4 \times 10^{-10}$ & $3.6 \times 10^{-10}$ & $3.4 \times 10^{-10}$ \\
\hline
\end{tabular}


Let Error $=\sqrt{\sum_{j=1}^{m}\left(V_{\text {caplet }}^{j}-\bar{V}_{\text {caplet }}\right)^{2} / m(m-1)}$ be the simulations error with classical Monte Carlo method and Error $_{s}=\sqrt{\sum_{j=1}^{m}\left(V_{\text {caplet }}^{j}(b)-\bar{V}_{\text {caplet }}(b)\right)^{2} / m(m-1)}(s=$ $1,2,3,4,5)$ be the $s$ th control variate Monte Carlo method as described above. Then Tables $1 \sim 3$ show that the control variate simulations have smaller errors than the classical Monte Carlo simulation errors. Moreover, the errors decrease when $\triangle t \rightarrow 0$ and $m \rightarrow \infty$.

Table 3 Simulation results for different control variates and simulation paths with $\Delta t=\frac{1}{3000}$

\begin{tabular}{ccccccc}
\hline$m$ & Error & Error1 & Error2 & Error3 & Error4 & Error5 \\
\hline $1 \times 10^{3}$ & $2.3 \times 10^{-6}$ & $1.1 \times 10^{-8}$ & $5.3 \times 10^{-9}$ & $5.4 \times 10^{-9}$ & $6.1 \times 10^{-9}$ & $5.2 \times 10^{-9}$ \\
$5 \times 10^{3}$ & $4.8 \times 10^{-7}$ & $2.1 \times 10^{-9}$ & $1.0 \times 10^{-9}$ & $1.1 \times 10^{-9}$ & $1.3 \times 10^{-9}$ & $1.6 \times 10^{-9}$ \\
$1 \times 10^{4}$ & $2.3 \times 10^{-7}$ & $1.0 \times 10^{-9}$ & $5.4 \times 10^{-10}$ & $5.3 \times 10^{-10}$ & $6.0 \times 10^{-10}$ & $5.1 \times 10^{-10}$ \\
$5 \times 10^{4}$ & $4.8 \times 10^{-8}$ & $2.1 \times 10^{-10}$ & $1.0 \times 10^{-10}$ & $1.1 \times 10^{-10}$ & $1.2 \times 10^{-10}$ & $1.6 \times 10^{-10}$ \\
\hline
\end{tabular}

Secondly, we present the simulation results for three caplets, the tenor structure is $0=T_{0}<$ $T_{1}<T_{2}<T_{3}<T_{4}<T_{5}$, and the terminal numeraire is $Q^{5}$. Let $R_{s}=\frac{\text { Error }}{\text { Error }_{s}}(s=1,2,3,4,5)$ be the variance reduction ratios. Then Table 4 shows the computation results of the variance reduction ratios for different control variates method described above and various simulation paths. Each $R_{i}$ is a 3 -dimension vector for there are 3 caplets.

Table 4 Simulation results for different control variates and simulation paths

\begin{tabular}{llllllllllllll}
\hline$m$ & & $10^{3}$ & \multicolumn{4}{c}{$5 \times 10^{3}$} & \multicolumn{3}{c}{$10^{4}$} & \multicolumn{3}{c}{$5 \times 10^{4}$} \\
\hline$R_{1}$ & 24.6 & 21.5 & 22.8 & 22.2 & 23.4 & 21.4 & 22.6 & 22.7 & 22.6 & 22.8 & 21.9 & 22.6 \\
$R_{2}$ & 24.3 & 22.0 & 22.8 & 22.1 & 23.7 & 21.3 & 22.7 & 22.8 & 22.5 & 22.7 & 22.8 & 22.5 \\
$R_{3}$ & 24.4 & 22.0 & 22.8 & 22.2 & 23.7 & 21.4 & 22.7 & 22.8 & 22.5 & 22.7 & 22.8 & 22.5 \\
$R_{4}$ & 24.4 & 22.0 & 22.8 & 22.2 & 23.6 & 21.3 & 22.8 & 22.6 & 22.5 & 22.9 & 21.8 & 21.6 \\
$R_{5}$ & 24.3 & 22.0 & 22.8 & 22.1 & 23.7 & 21.4 & 22.7 & 22.8 & 22.5 & 22.8 & 21.9 & 21.7 \\
\hline
\end{tabular}

Table 5 shows the computation results for different control variate as described above and different volatilities $\sigma$. We can see that the less $\sigma$ is, the greater variance reduction ratios are.

Table 5 Simulation results for different control variates and

\begin{tabular}{ccccccccccc}
\multicolumn{1}{c}{ volatilities $\sigma$ with $\triangle T=0.5, m=10^{3}, \triangle t=\frac{1}{30}$} \\
\hline \multicolumn{1}{c}{0.1} & \multicolumn{3}{c}{0.2} \\
\hline$R_{1}$ & 48.9 & 43.3 & 47.0 & 24.6 & 21.5 & 22.8 & 12.3 & 10.1 & 10.4 \\
$R_{2}$ & 48.6 & 44.3 & 47.2 & 24.3 & 22.0 & 22.8 & 12.1 & 10.4 & 10.4 \\
$R_{3}$ & 48.7 & 44.3 & 47.1 & 24.4 & 22.0 & 22.8 & 12.2 & 10.3 & 10.4 \\
$R_{4}$ & 49.0 & 44.2 & 47.0 & 24.4 & 22.0 & 22.8 & 12.2 & 10.4 & 10.4 \\
$R_{5}$ & 48.7 & 44.3 & 47.2 & 24.3 & 22.0 & 22.8 & 12.2 & 10.4 & 10.4 \\
\hline
\end{tabular}

Table 6 shows the simulation results for different control variates, and different mesh sizes $\triangle t$. We can see that the less $\triangle t$ is, the greater variance reduction ratios are. 
Table 6 Simulation results for different control variates and mesh size $\triangle t$ with $\triangle T=0.5, m=10^{3}, \sigma=0.2$

\begin{tabular}{llllllllll}
\hline$\triangle t$ & \multicolumn{3}{c}{$\frac{1}{30}$} & \multicolumn{3}{c}{$\frac{1}{60}$} & \multicolumn{5}{c}{$\frac{1}{120}$} \\
\hline$R_{1}$ & 24.6 & 21.5 & 22.8 & 30.5 & 31.5 & 30.4 & 38.8 & 40.3 & 41.6 \\
$R_{2}$ & 24.3 & 22.0 & 22.8 & 30.9 & 32.7 & 30.4 & 42.6 & 43.6 & 43.0 \\
$R_{3}$ & 24.4 & 22.0 & 22.8 & 31.0 & 32.7 & 30.4 & 42.6 & 43.7 & 43.0 \\
$R_{4}$ & 24.4 & 22.0 & 22.8 & 31.1 & 32.5 & 30.2 & 42.5 & 43.2 & 42.9 \\
$R_{5}$ & 24.3 & 22.0 & 22.8 & 30.9 & 32.7 & 30.4 & 42.6 & 43.6 & 43.0 \\
\hline
\end{tabular}

Table 7 shows the simulation results for different control variates and different tenor sizes $\triangle T$ with $\sigma=0.2, m=10^{3}, \triangle t=\frac{1}{30}$.

Table 7 Simulation results for different control variates and $\triangle T$

\begin{tabular}{cccccccccc}
$\triangle T$ & \multicolumn{3}{c}{0.5} & \multicolumn{3}{c}{0.25} & \multicolumn{3}{c}{1} \\
\hline$R_{1}$ & 24.6 & 21.5 & 22.8 & 34.8 & 31.3 & 33.2 & 16.5 & 13.6 & 15.0 \\
$R_{2}$ & 24.3 & 22.0 & 22.8 & 34.3 & 31.5 & 33.1 & 17.1 & 14.6 & 15.2 \\
$R_{3}$ & 24.4 & 22.0 & 22.8 & 34.3 & 31.5 & 33.1 & 17.4 & 14.6 & 15.3 \\
$R_{4}$ & 24.4 & 22.0 & 22.8 & 34.4 & 31.5 & 33.2 & 17.4 & 14.5 & 15.2 \\
$R_{5}$ & 24.3 & 22.0 & 22.8 & 34.3 & 31.5 & 33.1 & 17.2 & 14.6 & 15.2 \\
\hline
\end{tabular}

From Tables $4 \sim 7$, we find that the control variate methods show obvious variate reduction effects. While the less volatility $\sigma$ or mesh size $\Delta t$ or tenor size $\Delta T$ we take, the bigger variance reduction ratios $R$ we get. They also show that the Log-normal approximation, the linear optimal approximation exhibits a little better acceleration effect than other three.

We also compare the computation results for one-factor model with multi-factor model in Table 8, which shows that the variance reduction ratios of caplets based on one-factor model of LIBOR rate are greater than that based on multi-factor model of LIBOR.

Table 8 Simulation results for $\triangle T=0.5, \sigma=0.2, m=10^{3}, \triangle t=\frac{1}{30}$

\begin{tabular}{lllllll}
\hline & \multicolumn{3}{c}{ one-factor model } & \multicolumn{3}{c}{ multi-factor model } \\
\hline$R_{1}$ & 45.8 & 43.8 & 43.4 & 24.6 & 21.5 & 22.8 \\
$R_{2}$ & 45.6 & 46.7 & 45.7 & 24.3 & 22.0 & 22.8 \\
$R_{3}$ & 46.8 & 43.7 & 45.7 & 24.4 & 22.0 & 22.8 \\
$R_{4}$ & 46.8 & 43.2 & 45.5 & 24.4 & 22.0 & 22.8 \\
$R_{5}$ & 45.8 & 43.8 & 45.6 & 24.3 & 22.0 & 22.8 \\
\hline
\end{tabular}

Finally, we present the computational results in Table 9 for different numbers of caplets, which shows that the variance reduction ratios of caplets are evidently reduced when we take more caplets based on the approximation of LMM. 
Table 9 Simulation results for $\sigma=0.2, \triangle T=0.5, \triangle t=\frac{1}{30}, m=10^{3}$

\begin{tabular}{lccccccccc}
\hline & $V_{\text {caplet }_{1}}$ & $V_{\text {caplet }_{2}}$ & $V_{\text {caplet }_{3}}$ & $V_{\text {caplet }_{4}}$ & $V_{\text {caplet }_{5}}$ & $V_{\text {caplet }_{6}}$ & $V_{\text {caplet }_{7}}$ & $V_{\text {caplet }_{8}}$ & $V_{\text {caplet }_{9}}$ \\
\hline$R_{1}$ & 10.7453 & 9.9105 & 10.7491 & 11.9728 & 9.9338 & 10.8811 & 11.1514 & 10.1803 & 9.1079 \\
$R_{2}$ & 11.5201 & 11.4794 & 12.7718 & 13.1976 & 11.3506 & 11.3998 & 10.9209 & 10.2818 & 9.1657 \\
$R_{3}$ & 11.6520 & 11.5726 & 12.7744 & 13.3178 & 11.3892 & 11.4997 & 11.0540 & 10.1538 & 9.1707 \\
$R_{4}$ & 11.3826 & 11.4723 & 12.8434 & 13.1293 & 11.3545 & 11.2225 & 10.7508 & 10.2792 & 9.1654 \\
$R_{5}$ & 11.5601 & 11.5071 & 12.7860 & 13.2450 & 11.3336 & 11.4222 & 10.9557 & 10.2810 & 9.1653 \\
\hline
\end{tabular}

\section{Conclusion}

In this paper, we have proposed different control variates for Monte Carlo simulations, we illustrate the effects for different control variates of the underlying process for Monte Carlo simulation. The idea in this paper can also be extended to pricing other interest rate derivatives based on LIBOR market model, such as different kinds of Swaps, and so forth, by constructing control variate $H(\hat{L}(T))$, where $H(L(T))$ is the payoff of the interest rate derivative based on the LIBOR market model.

\section{References}

[1] Brace A, Gatarek D, Musiela M. The market model of interest rate dynamics. Mathematical Finance, 1997(7): 127-147.

[2] Miltersen K R, Sandmann K, Sondermann D. Cloesd-form solutions for term structure derivatives with log-nomal interest rates. Journal of Finance, 1997(70): 409-430.

[3] Glasserman P, Zhao X. Arbitrage-free discretization of lognormal forward LIBOR and swap rate models. Stochastic Finance, 2000(4): 35-68.

[4] Hull J, White A. Forward rate volatilities, Swap rate volatilities and the implementation of the LIBOR market model. Journal of Fixed Income, 2000(10): 46-62.

[5] Jackel P, Rebonato R. The link between Caplet and Swaption volatilities in a Brace-Gatarek-Musiela/ Jamshidian framwork: Approximation solution and empirical evidence. Journal of Computational Finance, 2003(6): 35-45.

[6] Hunter C, Jackel P, Joshi M. Getting the drift. Risk, 2001(14): 81-84.

[7] Kurbunmuradov O, Sabelfeld K, Schoenmakers J. Lognomal random field approximations to LIBOR market models. Journal of Computational Finance, 2002(6): 69-100.

[8] Joshi M, Stacey A. New and robust drift approximations for the LIBOR market model. Quantitative Finance, 2008(8): 427-434.

[9] Siopacha M, Teichmann J. Weak and strong Taylor methods for numerical solutions of stochastic differential equations. Quantitative Finance, 2011(11): 517-528.

[10] Papapantoleon A, Siopacha M. Strong Taylor approximation of SDEs and application to the Levy LIBOR model. Preprint, asXiv/0906.5581, 2009.

[11] Papapantoleon A, Skovmand D. Picard approximaton of stochastic differential equations and application to LIBOR models. Preprint, arXiv: 1007.3362, 2010.

[12] Pelsser A, Jong F D, Driessen J. LIBOR and Swap market models for the pricing of interest rate derivatives: An empirical comparison. Preprint, African Institute for Mathematical Sciences, 2004.

[13] Jamshidan F. LIBOR and swap market models and measures. Finance and Stochastics, 1997(1): $293-330$.

[14] Glasserman P. Monte Carlo methods in financial engineering. New York: Spinger, 2004. 